\title{
Measurement of the azimuthal correlation between the most forward jet and the scattered positron in deep-inelastic scattering at HERA
}

The H1 Collaboration

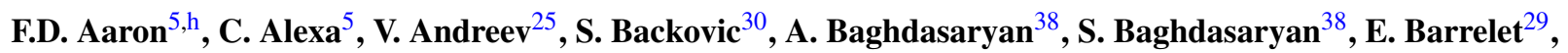
W. Bartel ${ }^{11}$, K. Begzsuren ${ }^{35}$, A. Belousov ${ }^{25}$, P. Belov ${ }^{11}$, J.C. Bizot ${ }^{27}$, V. Boudry ${ }^{28}$, I. Bozovic-Jelisavcic ${ }^{2}$, J. Bracinik ${ }^{3}$, G. Brandt $^{11}$, M. Brinkmann ${ }^{11}$, V. Brisson ${ }^{27}$, D. Britzger ${ }^{11}$, D. Bruncko ${ }^{16}$, A. Bunyatyan ${ }^{13,38}$, G. Buschhorn ${ }^{26, m}$, L. Bystritskaya ${ }^{24}$, A.J. Campbell ${ }^{11}$, K.B. Cantun Avila $^{22}$, F. Ceccopieri ${ }^{4}$, K. Cerny ${ }^{32}$, V. Cerny ${ }^{16, g}$, V. Chekelian ${ }^{26}$, J.G. Contreras ${ }^{22}$, J.A. Coughlan ${ }^{6}$, J. Cvach ${ }^{31}$, J.B. Dainton ${ }^{18}$, K. Daum ${ }^{37, \text { c }}$, B. Delcourt ${ }^{27}$, J. Delvax ${ }^{4}$, E.A. De Wolf ${ }^{4}$, C. Diaconu ${ }^{21}$, M. Dobre ${ }^{12, j, k}$, V. Dodonov ${ }^{13}$, A. Dossanov ${ }^{26}$, A. Dubak ${ }^{30, f}$, G. Eckerlin $^{11}$, S. Egli ${ }^{36}$, A. Eliseev $^{25}$, E. Elsen ${ }^{11}$, L. Favart ${ }^{4}$, A. Fedotov ${ }^{24}$, R. Felst ${ }^{11}$, J. Feltesse ${ }^{10}$, J. Ferencei ${ }^{16}$, D.-J. Fischer ${ }^{11}$, M. Fleischer ${ }^{11}$, A. Fomenko ${ }^{25}$, E. Gabathuler ${ }^{18}$, J. Gayler ${ }^{11}$, S. Ghazaryan ${ }^{11}$, A. Glazov ${ }^{11}$, L. Goerlich ${ }^{7}$, N. Gogitidze ${ }^{25}$, M. Gouzevitch ${ }^{11, e}$, C. Grab ${ }^{40}$, A. Grebenyuk ${ }^{11}$, T. Greenshaw ${ }^{18}$, B.R. Grell ${ }^{11}$, G. Grindhammer ${ }^{26}$, S. Habib ${ }^{11}$, D. Haidt ${ }^{11}$, C. Helebrant ${ }^{11}$, R.C.W. Henderson ${ }^{17}$, E. Hennekemper ${ }^{15}$, H. Henschel ${ }^{39}$, M. Herbst ${ }^{15}$, G. Herrera ${ }^{23}$, M. Hildebrandt ${ }^{36}$, K.H. Hiller ${ }^{39}$, D. Hoffmann ${ }^{21}$, R. Horisberger ${ }^{36}$, T. Hreus ${ }^{4, d}$, F. Huber ${ }^{14}$, M. Jacquet ${ }^{27}$, X. Janssen ${ }^{4}$, L. Jönsson ${ }^{20}$, H. Jung ${ }^{4,11,1}$, M. Kapichine ${ }^{9}$, I.R. Kenyon ${ }^{3}$, C. Kiesling ${ }^{26}$, M. Klein ${ }^{18}$, C. Kleinwort ${ }^{11}$, T. Kluge ${ }^{18}$, R. Kogler $^{11}$, P. Kostka ${ }^{39}$, M. Kraemer ${ }^{11}$, J. Kretzschmar ${ }^{18}$, K. Krüger ${ }^{15}$, a M.P.J. Landon ${ }^{19}$, W. Lange ${ }^{39}$, G. Laštovička-Medin ${ }^{30}$, P. Laycock ${ }^{18}$, A. Lebedev ${ }^{25}$, V. Lendermann ${ }^{15}$, S. Levonian ${ }^{11}$, K. Lipka ${ }^{11, j}$, B. List ${ }^{11}$, J. List $^{11}$, R. Lopez-Fernandez ${ }^{23}$, V. Lubimov ${ }^{24}$, A. Makankine ${ }^{9}$, E. Malinovski ${ }^{25}$, P. Marage ${ }^{4}$, H.-U. Martyn ${ }^{1}$,

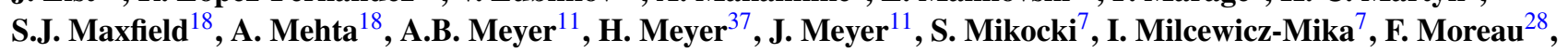
A. Morozov ${ }^{9}$, J.V. Morris ${ }^{6}$, M. Mudrinic ${ }^{2}$, K. Müller ${ }^{41}$, Th. Naumann ${ }^{39}$, P.R. Newman ${ }^{3}$, C. Niebuhr ${ }^{11}$, D. Nikitin ${ }^{9}$, G. Nowak ${ }^{7}$, K. Nowak ${ }^{11}$, J.E. Olsson ${ }^{11}$, D. Ozerov ${ }^{24}$, P. Pahl ${ }^{11}$, V. Palichik ${ }^{9}$, I. Panagoulias ${ }^{11, b, y}$, M. Pandurovic ${ }^{2}$,

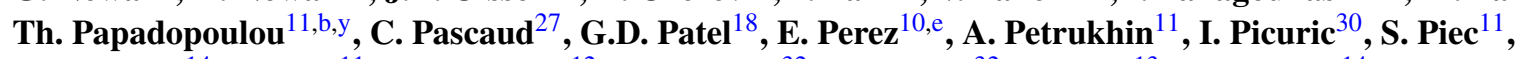
H. Pirumov ${ }^{14}$, D. Pitzl ${ }^{11}$, R. Plačakytè ${ }^{12}$, B. Pokorny ${ }^{32}$, R. Polifka ${ }^{32}$, B. Povh ${ }^{13}$, V. Radescu ${ }^{14}$, N. Raicevic ${ }^{30}$, T. Ravdandorj ${ }^{35}$, P. Reimer $^{31}$, E. Rizvi ${ }^{19}$, P. Robmann ${ }^{41}$, R. Roosen ${ }^{4}$, A. Rostovtsev ${ }^{24}$, M. Rotaru ${ }^{5}$, J.E. Ruiz Tabasco ${ }^{22}$, S. Rusakov ${ }^{25}$, D. Š́lek ${ }^{32}$, D.P.C. Sankey ${ }^{6}$, M. Sauter ${ }^{14}$, E. Sauvan ${ }^{21}$, S. Schmitt ${ }^{11}$, L. Schoeffel ${ }^{10}$, A. Schöning ${ }^{14}$, H.-C. Schultz-Coulon ${ }^{15}$, F. Sefkow ${ }^{11}$, L.N. Shtarkov ${ }^{25}$, S. Shushkevich ${ }^{11}$, T. Sloan ${ }^{17}$, I. Smiljanic ${ }^{2}$, Y. Soloviev ${ }^{25}$, P. Sopicki ${ }^{7}$, D. South ${ }^{11}$, V. Spaskov ${ }^{9}$, A. Specka ${ }^{28}$, Z. Staykova ${ }^{4}$, M. Steder ${ }^{11}$, B. Stella ${ }^{33}$, G. Stoicea $^{5}$, U. Straumann ${ }^{41}$, T. Sykora ${ }^{4,32}$, P.D. Thompson ${ }^{3}$, T.H. Tran ${ }^{27}$, D. Traynor ${ }^{19}$, P. Truöll1 ${ }^{41}$ I. Tsakov ${ }^{34}$, B. Tseepeldorj j5, , J. Turnau ${ }^{7}$, A. Valkárová ${ }^{32}$, C. Vallée ${ }^{21}$, P. Van Mechelen ${ }^{4}$, Y. Vazdik $^{25}$, D. Wegener ${ }^{8}$,

E. Wünsch ${ }^{11}$, J. Žáček ${ }^{32}$, J. Zálešák ${ }^{31}$, Z. Zhang ${ }^{27}$, A. Zhokin ${ }^{24}$, H. Zohrabyan ${ }^{38}$, F. Zomer ${ }^{27}$

${ }^{1}$ I. Physikalisches Institut der RWTH, Aachen, Germany

${ }^{2}$ Vinca Institute of Nuclear Sciences, University of Belgrade, 1100 Belgrade, Serbia

${ }^{3}$ School of Physics and Astronomy, University of Birmingham, Birmingham, UK $^{\circ}$

${ }_{5}^{4}$ Inter-University Institute for High Energies ULB-VUB, Brussels and Universiteit Antwerpen, Antwerpen, Belgium ${ }^{\mathrm{p}}$

${ }^{5}$ National Institute for Physics and Nuclear Engineering (NIPNE), Bucharest, Romania ${ }^{z}$

${ }^{6}$ Rutherford Appleton Laboratory, Chilton, Didcot, $\mathrm{UK}^{\mathrm{O}}$

${ }^{7}$ Institute for Nuclear Physics, Cracow, Poland ${ }^{9}$

${ }^{8}$ Institut für Physik, TU Dortmund, Dortmund, Germany ${ }^{\mathrm{n}}$

${ }^{9}$ Joint Institute for Nuclear Research, Dubna, Russia

${ }^{10}$ CEA, DSM/Irfu, CE-Saclay, Gif-sur-Yvette, France

${ }^{11}$ DESY, Hamburg, Germany

${ }^{12}$ Institut für Experimentalphysik, Universität Hamburg, Hamburg, Germany ${ }^{\text {n }}$

${ }^{13}$ Max-Planck-Institut für Kernphysik, Heidelberg, Germany

${ }^{14}$ Physikalisches Institut, Universität Heidelberg, Heidelberg, Germany ${ }^{\mathrm{n}}$

${ }^{15}$ Kirchhoff-Institut für Physik, Universität Heidelberg, Heidelberg, Germany ${ }^{\mathrm{n}}$

${ }^{16}$ Institute of Experimental Physics, Slovak Academy of Sciences, Košice, Slovak Republic ${ }^{\mathrm{s}}$

${ }^{17}$ Department of Physics, University of Lancaster, Lancaster, UK ${ }^{\mathrm{O}}$ 
${ }^{18}$ Department of Physics, University of Liverpool, Liverpool, $\mathrm{UK}^{\mathrm{o}}$

${ }^{19}$ Queen Mary and Westfield College, London, UK ${ }^{\circ}$

${ }^{20}$ Physics Department, University of Lund, Lund, Sweden ${ }^{\mathrm{t}}$

${ }^{21}$ CPPM, Aix-Marseille Univ, CNRS/IN2P3, 13288 Marseille, France

${ }^{22}$ Departamento de Fisica Aplicada, CINVESTAV, Mérida, Yucatán, Mexicow

${ }^{23}$ Departamento de Fisica, CINVESTAV IPN, México City, Mexico ${ }^{\mathrm{w}}$

${ }^{24}$ Institute for Theoretical and Experimental Physics, Moscow, Russia ${ }^{\mathrm{x}}$

${ }^{25}$ Lebedev Physical Institute, Moscow, Russia ${ }^{\mathrm{r}}$

${ }^{26}$ Max-Planck-Institut für Physik, München, Germany

${ }^{27}$ LAL, Université Paris-Sud, CNRS/IN2P3, Orsay, France

${ }^{28}$ LLR, Ecole Polytechnique, CNRS/IN2P3, Palaiseau, France

${ }^{29}$ LPNHE, Université Pierre et Marie Curie Paris 6, Université Denis Diderot Paris 7, CNRS/IN2P3, Paris, France

${ }^{30}$ Faculty of Science, University of Montenegro, Podgorica, Montenegro ${ }^{\text {aa }}$

${ }^{31}$ Institute of Physics, Academy of Sciences of the Czech Republic, Praha, Czech Republic ${ }^{u}$

${ }^{32}$ Faculty of Mathematics and Physics, Charles University, Praha, Czech Republic ${ }^{\mathrm{u}}$

${ }^{33}$ Dipartimento di Fisica, Università di Roma Tre and INFN Roma 3, Roma, Italy

${ }^{34}$ Institute for Nuclear Research and Nuclear Energy, Sofia, Bulgaria ${ }^{\mathrm{r}}$

${ }^{35}$ Institute of Physics and Technology of the Mongolian Academy of Sciences, Ulaanbaatar, Mongolia

${ }^{36}$ Paul Scherrer Institut, Villigen, Switzerland

${ }^{37}$ Fachbereich C, Universität Wuppertal, Wuppertal, Germany

${ }^{38}$ Yerevan Physics Institute, Yerevan, Armenia

${ }^{39}$ DESY, Zeuthen, Germany

${ }^{40}$ Institut für Teilchenphysik, ETH, Zürich, Switzerland ${ }^{\mathrm{V}}$

${ }^{41}$ Physik-Institut der Universität Zürich, Zürich, Switzerland ${ }^{v}$

Received: 17 November 2011 / Revised: 27 January 2012 / Published online: 6 March 2012

(C) The Author(s) 2012. This article is published with open access at Springerlink.com

Abstract Deep-inelastic positron-proton scattering events at low photon virtuality, $Q^{2}$, with a forward jet, produced at small angles with respect to the proton beam, are measured with the $\mathrm{H} 1$ detector at HERA. A subsample of events with an additional jet in the central region is also studied. For both samples, differential cross sections and normalised distributions are measured as a function of the az-

a e-mail: krueger@mail.desy.de

${ }^{\mathrm{b}}$ Also at Physics Department, National Technical University, Zografou Campus, GR-15773 Athens, Greece.

${ }^{c}$ Also at Rechenzentrum, Universität Wuppertal, Wuppertal, Germany.

${ }^{\mathrm{d}}$ Also at University of P.J. Šafárik, Košice, Slovak Republic.

e Also at CERN, Geneva, Switzerland.

${ }^{\mathrm{f}}$ Also at Max-Planck-Institut für Physik, München, Germany.

${ }^{\mathrm{g}}$ Also at Comenius University, Bratislava, Slovak Republic.

${ }^{h}$ Also at Faculty of Physics, University of Bucharest, Bucharest, Romania.

${ }^{\mathrm{i}}$ Also at Ulaanbaatar University, Ulaanbaatar, Mongolia.

${ }^{j}$ Supported by the Initiative and Networking Fund of the Helmholtz Association (HGF) under the contract VH-NG-401.

${ }^{\mathrm{k}}$ Absent on leave from NIPNE-HH, Bucharest, Romania.

${ }^{l}$ On leave of absence at CERN, Geneva, Switzerland.

m Deceased.

${ }^{\mathrm{n}}$ Supported by the Bundesministerium für Bildung und Forschung, FRG, under contract numbers 05H09GUF, 05H09VHC, 05H09VHF, 05H16PEA. imuthal angle difference, $\Delta \phi$, between the forward jet and the scattered positron in bins of the rapidity distance, $Y$, between them. The data are compared to predictions of Monte Carlo generators based on different evolution approaches as well as to next-to-leading order calculations in order to test the sensitivity to QCD evolution mechanisms.

${ }^{\circ}$ Supported by the UK Science and Technology Facilities Council, and formerly by the UK Particle Physics and Astronomy Research Council. pSupported by FNRS-FWO-Vlaanderen, IISN-IIKW and IWT and by Interuniversity Attraction Poles Programme, Belgian Science Policy.

q Partially Supported by Polish Ministry of Science and Higher Education, grant DPN/N168/DESY/2009.

${ }^{\mathrm{r}}$ Supported by the Deutsche Forschungsgemeinschaft.

${ }^{\text {s }}$ Supported by VEGA SR grant no. 2/7062/27.

${ }^{\mathrm{t}}$ Supported by the Swedish Natural Science Research Council.

"Supported by the Ministry of Education of the Czech Republic under the projects LC527, INGO-LA09042 and MSM0021620859.

${ }^{\mathrm{v}}$ Supported by the Swiss National Science Foundation.

${ }^{\mathrm{w}}$ Supported by CONACYT, México, grant 48778-F.

${ }^{\mathrm{x}}$ Russian Foundation for Basic Research (RFBR), grant no. 1329.2008.2 and Rosatom.

${ }^{\mathrm{y}}$ This project is co-funded by the European Social Fund (75\%) and National Resources (25\%) — (EPEAEK II) —PYTHAGORAS II.

${ }^{\mathrm{z}}$ Supported by the Romanian National Authority for Scientific Research under the contract PN 09370101.

${ }^{a}$ Partially Supported by Ministry of Science of Montenegro, no. 051/3-3352. 


\section{Introduction}

Measurements of the hadronic final state in deep-inelastic lepton-proton scattering (DIS) test Quantum Chromodynamics (QCD), the theory of the strong force. At moderate negative four-momentum transfers squared, $Q^{2}$, of a few $\mathrm{GeV}^{2}$, the HERA ep collider has extended the available kinematic range for deep-inelastic scattering to regions of small Bjorken- $x \simeq 10^{-4}$. This is the region of high parton densities in the proton, dominated by gluons and sea quarks. Figure 1 shows a generic diagram for parton evolution in a DIS process at low $\mathrm{x}$, in which a gluon from the proton induces a QCD cascade before an interaction with the virtual photon.

At the large $\gamma^{*} p$ centre-of-mass energy available at small $x$, a transition is expected from parton cascades ordered in transverse momentum, described by the DokshitzerGribov-Lipatov-Altarelli-Parisi (DGLAP) evolution equations [1-5], to cascades unordered in transverse momentum, described by the Balitsky-Fadin-Kuraev-Lipatov (BFKL) approach [6-8]. In the DGLAP approximation, the struck quark originates from a parton cascade ordered in virtualities of the propagator partons. At low $x$ this implies a strong ordering in transverse momentum, $k_{T}$, of the emitted partons, measured with respect to the proton direction. In the BFKL approach there is no ordering in $k_{T}$ of the partons along the ladder. Compared to the DGLAP scheme more gluons with sizable transverse momentum are emitted near the proton direction. For this reason energetic jets of high transverse momentum produced close to the proton direction in the laboratory frame, referred to as the forward region, are considered to be especially sensitive to QCD dynamics at

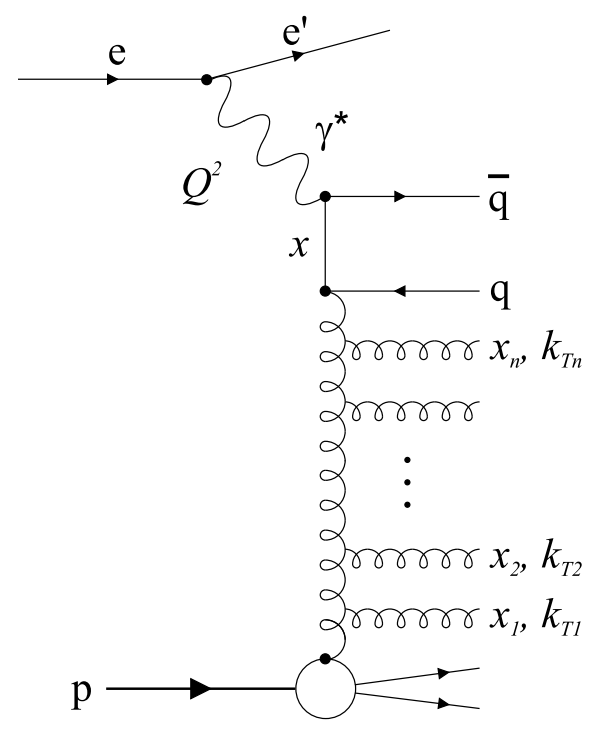

Fig. 1 Generic diagram for deep-inelastic ep scattering at small $x$. A gluon cascade evolves between the quark box, attached to the virtual photon, and the proton. The gluon longitudinal momentum fractions and transverse momenta are labeled $x_{i}$ and $k_{T i}$, respectively low $x$ [9]. Forward jet production was measured previously by the $\mathrm{H} 1$ and ZEUS collaborations. In these measurements as well as in the present one, the requirements on the forward jet and the phase space were chosen in such a way that the standard DGLAP evolution is suppressed and the effects of BFKL dynamics are enhanced. Preference for models which employ QCD evolution non-ordered in transverse momentum was observed [10-16].

One of the observables suggested to be sensitive to BFKL dynamics [17] is the azimuthal angle difference, $\Delta \phi$, between the forward jet and the scattered electron, defined in the laboratory frame. In the Quark Parton Model (QPM) process $e+q \rightarrow e+q$, the simple two-body kinematics constrain the scattered electron and the jet to be produced back-to-back, and thus predict $\Delta \phi=\pi$ at the parton-level. Hadronisation effects induce some smearing to this partonlevel prediction. Inclusion of higher order processes partially decorrelates the jet from the electron. As a consequence, for evolution schemes without ordering in transverse momentum, the decorrelation is expected to increase with electron-jet rapidity distance, $Y$, since the phase space for additional parton emissions increases. The calculations employing the BFKL approach to next-to-leading order accuracy, indeed, predict an increase of the azimuthal angle decorrelation with the electron-jet rapidity distance [18].

This paper presents a study of low $x$ DIS interactions in which high transverse momentum jets are produced in the forward region. The forward jet cross sections and normalised distributions are measured as a function of the azimuthal angle difference, $\Delta \phi$, in three bins of the rapidity separation, $Y$, between the positron and the forward jet. The forward jet cross section as a function of $Y$ is also measured. Moreover, the measurements of the azimuthal correlations in $\Delta \phi$ are performed using a subsample in which an additional central jet is required. In comparison with the forward jet sample, this subsample is expected to contain a higher fraction of forward jets from additional gluon emissions.

The data set used for the analysis was collected with the $\mathrm{H} 1$ detector in the year 2000, when positrons and protons collided with energies of $27.6 \mathrm{GeV}$ and $920 \mathrm{GeV}$, respectively, corresponding to a centre-of-mass energy of $\sqrt{s}=319 \mathrm{GeV}$. The integrated luminosity of the data set is $38.2 \mathrm{pb}^{-1}$, which is about fourteen times larger than that used in the previous measurement of the azimuthal decorrelation of forward jets [11].

\section{QCD calculations}

The measurements presented here are compared with predictions of Monte Carlo (MC) programs and perturbative QCD calculations at next-to-leading order (NLO). The MC programs use first-order QCD matrix elements and model higher order terms by parton showers in the leading loga- 
rithm approximation or by quasi-classical gluon radiation from colour dipoles. Three MC event generators, which adopt different QCD based approaches to model the parton cascade, are used.

- RAPGAP [19] matches first order QCD matrix elements to DGLAP based leading-log parton showers with $k_{T}$ ordering. The factorisation and renormalisation scales are set to $\mu_{f}=\mu_{r}=\sqrt{Q^{2}+p_{\mathrm{T}}^{2}}$, where $p_{\mathrm{T}}$ is the transverse momentum of the two outgoing hard partons in the centreof-mass of the hard subsystem. Predictions of RAPGAP are labeled DGLAP in the figures.

- DJANGOH [20] with ARIADNE includes an implementation of the Colour Dipole Model (CDM) [21], which has as its basic construct a colour dipole formed by the struck quark and the proton remnant. Subsequent parton emissions originate from a chain of independently radiating dipoles formed by the emitted gluons. In this approach the transverse momenta of emitted gluons perform a random walk such that CDM provides a BFKL-like approach. The leading order partonic final state is corrected to exactly reproduce the $O\left(\alpha_{S}\right)$ matrix elements. The simulation of DJANGOH/ARIADNE uses a set of colour dipole parameters tuned to describe measurements of the hadronic final state in DIS at HERA [22]. The DJANGOH/ARIADNE predictions are referred to as CDM in the following.

- CASCADE $[23,24]$ implements the Ciafaloni-CataniFiorani-Marchesini (CCFM) evolution [25-27] which aims to unify the DGLAP and BFKL approaches. It introduces angular ordering of emissions to implement gluon coherence effects and thus in the high energy limit, the CCFM evolution equation is almost equivalent to the BFKL approach, while reproducing the DGLAP equations for large $x$ and high $Q^{2}$. CASCADE uses off-shell leading order QCD matrix elements, supplemented with gluon emissions based on the CCFM evolution equation, requiring an unintegrated gluon density function (uPDF), which takes the transverse momenta of the propagators into account. In this paper two different uPDF sets are used: set A0 [28] with only singular terms of the gluon splitting function and J2003-set 2 [29] including also nonsingular terms, labeled set 2 in the figures. These parameterisations for the unintegrated gluon density were obtained using the CCFM evolution equation to describe the structure function $F_{2}\left(x, Q^{2}\right)$ as measured by $\mathrm{H} 1[30,31]$ and ZEUS [32, 33]. Predictions of CASCADE are labeled CCFM in the figures.

To perform the hadronisation step, all of the above models use the Lund string fragmentation scheme, as implemented in JETSET [34] in the case of DJANGOH/ARIADNE and in PYTHIA [35] for RAPGAP and CASCADE, using a tuning based on LEP $e^{+} e^{-}$data [36]. The RAPGAP and DJANGOH/ARIADNE predictions are calculated using the HERAPDF1.0 [37] set of parton distribution functions (PDF).

The RAPGAP and DJANGOH/ARIADNE programs are interfaced with HERACLES [38], which allows the simulation of QED-radiative effects. These MC models are used to simulate detector effects in order to determine the acceptance and efficiency for selected forward jet events in DIS. Generated events are passed through a GEANT [39] based simulation of the $\mathrm{H} 1$ apparatus, which takes into account the running conditions of the data taking. Simulated events are reconstructed and analysed using the same program chain as is used for the data.

The measurements of azimuthal correlations are also compared to the fixed order NLO DGLAP predictions of NLOJET++ [40]. The NLOJET++ program is used here to calculate dijet production at parton level in DIS at $\operatorname{NLO}\left(\alpha_{S}^{2}\right)$ accuracy. It should be noted that the jet search is performed on partons in the Breit frame (see Sect. 3.2), and therefore the events contain at least one jet in addition to the forward jet. The renormalisation and factorisation scales are defined for each event and are set to $\mu_{r}=\mu_{f}=\sqrt{\left(P_{\mathrm{T}, \mathrm{sc}}^{2}+Q^{2}\right) / 2}$, where $P_{\mathrm{T}, \mathrm{sc}}$ is the transverse momentum of the forward jet in the forward jet sample or the average transverse momentum of the forward and central jet in the sample with the additional central jet. The NLO calculations are performed using the CTEQ6.6 [41] parameterisation of the parton distributions in the proton. The NLOJET++ parton level cross sections are corrected for hadronisation effects using the RAPGAP model. The correction factors for hadronisation are estimated bin-by-bin by calculating the ratio between the cross section for jets reconstructed from stable hadrons (hadron level) and the parton level cross section. The correction factors for hadronisation are in the range from 0.90 to 1.08 , increasing with rapidity distance $Y$. The uncertainty of the NLOJET++ predictions due to missing higher orders is estimated by applying a factor 2 or $1 / 2$ to the renormalisation and factorisation scales simultaneously.

\section{Experimental method}

\section{$3.1 \mathrm{H} 1$ detector}

A detailed description of the $\mathrm{H} 1$ detector can be found elsewhere [42-44]. The components of the detector which are most relevant for this analysis are briefly described below. The origin of the $\mathrm{H} 1$ coordinate system is the nominal ep interaction point. The direction of the proton beam defines the positive $z$-axis. Transverse momenta, $p_{\mathrm{T}}$, and polar angles, $\theta$, of all particles are defined with respect to this direction. The azimuthal angle, $\phi$, defines the particle direction in the transverse plane. The pseudorapidity is given by $\eta=-\ln$ $(\tan \theta / 2)$.

The $e p$ interaction region is surrounded by the central tracking detector (CTD) consisting of two large concentric 
drift chambers, operated inside a $1.16 \mathrm{~T}$ solenoidal magnetic field. Charged particles are measured in the angular range $20^{\circ}<\theta<160^{\circ}$ with a transverse momentum resolution of $\sigma_{p_{\mathrm{T}}} / p_{\mathrm{T}} \approx 0.005 \cdot p_{\mathrm{T}}[\mathrm{GeV}] \oplus 0.015$. Information from the CTD is used to trigger events, to locate the event vertex and contributes to the reconstruction of the hadronic final state.

A highly segmented liquid argon (LAr) calorimeter is used to measure the hadronic final state. It covers the range of the polar angle $4^{\circ}<\theta<154^{\circ}$ and offers full azimuthal coverage. The LAr calorimeter consists of an electromagnetic section with lead absorbers and a hadronic section with steel absorbers. The total depth of both sections varies between 4.5 and 8 interaction lengths in the region $4^{\circ}<\theta<128^{\circ}$ and between 20 and 30 radiation lengths in the region $4^{\circ}<\theta<154^{\circ}$ increasing towards the forward direction. Test beam measurements of the LAr calorimeter modules showed an energy resolution of $\sigma_{E} / E \approx 0.50 / \sqrt{E[\mathrm{GeV}]} \oplus 0.02$ for charged pions [45] and of $\sigma_{E} / E \approx 0.12 / \sqrt{E[\mathrm{GeV}]} \oplus 0.01$ for electrons [46].

A lead/scintillating fiber calorimeter (SpaCal) [44] covers the region $153^{\circ}<\theta<177.5^{\circ}$. It has an electromagnetic and a hadronic section and is used to measure the scattered positron and the backward hadronic energy flow. The energy resolution, determined from test beam measurements [47], is $\sigma_{E} / E \approx 0.07 / \sqrt{E[\mathrm{GeV}]} \oplus 0.01$ for electrons. The precision of the measurement of the polar angle of the positron, improved using the backward drift chamber (BDC) situated in front of the SpaCal calorimeter, is $1 \mathrm{mrad}$.

The luminosity determination is based on the measurement of the Bethe-Heitler process ep $\rightarrow$ ep $\gamma$ where the photon is detected in a calorimeter located at $z=-103 \mathrm{~m}$ downstream of the interaction region in the positron beam direction.

\subsection{Event selection}

DIS events are selected using triggers based on electromagnetic energy deposits in the SpaCal calorimeter and the presence of charged particle tracks in the central tracker. The trigger efficiency is determined using independently triggered data. For DIS events with a forward jet, the trigger efficiency lies between $60 \%$ and $80 \%$, and for the 'forward and central jet' topology it is at the level of $80 \%$.
The data set is restricted in inelasticity, $y$, photon virtuality, $Q^{2}$, and $x: 0.1<y<0.7,5<Q^{2}<85 \mathrm{GeV}^{2}$, $0.0001<x<0.004$. In this analysis these variables are determined from measurements of the scattered positron energy and its polar angle and from the incident positron beam energy. This phase space is chosen to ensure that the DIS kinematics are well determined and to reduce the background from photoproduction.

The background from photoproduction and from events with large initial-state QED radiation is further reduced by requiring $35<\Sigma_{i}\left(E_{i}-p_{z, i}\right)<70 \mathrm{GeV}$. Here $E_{i}$ and $p_{z, i}$ are the energy and longitudinal momentum of a particle $i$, respectively, and the sum extends over all detected particles in the event. Energy-momentum conservation requires that $\Sigma_{i}\left(E_{i}-p_{z, i}\right)=2 \cdot E_{e}^{0}$, where $E_{e}^{0}$ is the positron beam energy. Jets are identified from combined calorimeter and track objects [48] using the $k_{T}$ cluster algorithm in the longitudinally invariant inclusive mode $[49,50]$ applied in the Breit frame. The reconstructed jets are then boosted to the laboratory frame.

The measurements of forward jets are restricted to the phase space region where the transverse momentum of the jet is approximately equal to the photon virtuality, $P_{\mathrm{T}, \mathrm{fwdjet}}^{2} \approx Q^{2}$. This condition suppresses the contribution of $k_{T}$-ordered DGLAP cascades with respect to processes unordered in $k_{T}$ [9]. The selection of forward jets with a large fraction of the proton energy, $x_{\mathrm{fwdjet}} \equiv E_{\mathrm{fwdjet}} / E_{p}$, such that $x_{\mathrm{fwdjet}} \gg x$, enhances the phase space for BFKL evolution with gluon cascades strongly ordered in fractional longitudinal momentum. The above conditions are fulfilled by the requirement that the analysed sample contains at least one forward jet which satisfies the following criteria in the laboratory frame: $P_{\mathrm{T} \text {,fwdjet }}>6 \mathrm{GeV}, 1.73<\eta_{\text {fwdjet }}<2.79$, $x_{\text {fwdjet }}>0.035$ and $0.5<P_{\mathrm{T} \text {,fwdjet }}^{2} / Q^{2}<6$. Here $\eta_{\text {fwdjet }}$ is the pseudorapidity of the forward jet. If there is more than one jet fulfilling the above requirements, the jet with the largest pseudorapidity is chosen. The upper cut on $P_{\mathrm{T}, \mathrm{fwdjet}}^{2} / Q^{2}$ is chosen so large in order to reduce the contributions of migrations from outside of the analysis phase space, which are due to the limited resolution of the $P_{\mathrm{T} \text {,fwdjet }}$ measurement.

The 'forward and central jet' subsample is selected by requiring an additional jet in the central region of the lab-

Table 1 Summary of cuts defining the DIS phase space, the forward jet and the central jet selection. If more than one forward jet is found, the jet with the largest $\eta_{\mathrm{fwdjet}}$ is chosen. If there is more than one central jet, the one with the smallest $\eta_{\text {cenjet }}$ is selected

\begin{tabular}{lll}
\hline DIS selection & Forward jets & Central jets \\
\hline $0.1<y<0.7$ & $1.73<\eta_{\mathrm{fwdjet}}<2.79$ & $-1<\eta_{\text {cenjet }}<1$ \\
$5<Q^{2}<85 \mathrm{GeV}^{2}$ & $P_{\mathrm{T}, \text { fwdjet }}>6 \mathrm{GeV}$ & $P_{\mathrm{T}, \mathrm{cenjet}}>4 \mathrm{GeV}$ \\
$0.0001<x<0.004$ & $x_{\mathrm{fwdjet}}>0.035$ & $\Delta \eta=\eta_{\mathrm{fwdjet}}-\eta_{\text {cenjet }}>2$ \\
& $0.5<P_{\mathrm{T}, \text { fwdjet }}^{2} / Q^{2}<6$ & \\
\hline
\end{tabular}


oratory frame. This jet is required to have a transverse momentum $P_{\mathrm{T} \text {,cenjet }}>4 \mathrm{GeV}$ and to lie in the pseudorapidity region $-1<\eta_{\text {cenjet }}<1$. The central jet must have a large rapidity separation from the most forward jet $\Delta \eta=$ $\left(\eta_{\mathrm{fwdjet}}-\eta_{\text {cenjet }}\right)>2$. This condition enhances the phase space for additional parton emissions between the two jets. If there is more than one central jet, the one with the smallest $\eta_{\text {cenjet }}$ is chosen.

A summary of the selection cuts, defining the DIS phase space for the measurement, the forward jet sample and the subsample with an additional central jet, is provided in Table 1 . With these requirements 13736 (8871) events are selected for the forward jet (forward and central jet) analysis.

\subsection{Cross section determination}

In this measurement, in addition to migrations between bins inside the measurement phase space, there are considerable migrations from outside the analysis phase space. This is taken into account in the calculation of the cross section corrected to the hadron level:

$\sigma_{i}=\frac{N_{i}^{\text {data }}-N_{i}^{\text {out }}}{\epsilon_{i} \cdot \mathcal{L}}$.

Here $N_{i}^{\text {data }}$ is the number of observed events in bin $i, N_{i}^{\text {out }}$ is the number of events from outside the measurement phase space reconstructed in bin $i$, and $\epsilon_{i}$ is the efficiency in bin $i$. $\mathcal{L}$ is the total integrated luminosity. $N_{i}^{\text {out }}$ and $\epsilon_{i}$ are estimated using MC simulations. The purities ${ }^{1}$ in bins of the measured cross sections, as determined from the MC simulations, are at the level of $80 \%$.

The efficiency factors $\epsilon_{i}$ are calculated according to the formula:

$\epsilon_{i}=\frac{N_{i}^{\mathrm{det}}-N_{i}^{\mathrm{out}}}{N_{i}^{\mathrm{had}}}$,

where $N_{i}^{\text {det }}$ and $N_{i}^{\text {had }}$ are the numbers of events in bin $i$ at the detector and at the hadron level, respectively. For this approach to be valid, the shape of the distributions of all variables on which phase space cuts are applied have to be well described by the MC simulations also in the phase space extended beyond these cuts. This requirement is found to be satisfied by both models considered here.

The efficiency factors are calculated as the ratio of the model prediction at the detector level for a radiative MC and at the hadron level for a non-radiative MC, i.e. the data are also corrected for QED radiative effects. The efficiency factors are taken as the average of the factors estimated by the RAPGAP and DJANGOH/ARIADNE models. The uncertainty of the efficiency factors is taken to be half of the

\footnotetext{
${ }^{1}$ The purity is defined as the ratio of the number of events generated and reconstructed in the bin to the number of events originating from the phase space of the analysis and reconstructed in that bin.
}

difference between the factors calculated using the two MC models and is included in the systematic error.

\subsection{Systematic uncertainties}

The following sources of systematic uncertainties are considered:

- The model dependence of the bin-by-bin efficiency factors, $\epsilon_{i}$, leads to systematic uncertainties between $2 \%$ and $6 \%$ for the measured cross sections.

- The LAr hadronic energy scale uncertainty of $4 \%$ for this analysis gives rise to the dominant uncertainty of $7 \%$ to $12 \%$ for the measured cross sections.

- The uncertainty on the electromagnetic energy scale of the $\mathrm{SpaCal}$ of $1 \%$ results in an uncertainty of the measured cross sections below $3 \%$.

- The uncertainty on the polar angle measurement of the scattered positron of $1 \mathrm{mrad}$ has a negligible effect on the cross section measurements.

- The uncertainty on the determination of the trigger efficiency from the data, using independent trigger samples, leads to an uncertainty between $2 \%$ and $4 \%$ on the cross section measurements.

- The measurement of the integrated luminosity is accurate to within $1.5 \%$.

The total systematic uncertainty, adding all above mentioned individual contributions quadratically, amounts on average to $11-12 \%$ for the measured cross sections.

\section{Results}

The forward jet cross sections and their uncertainties are given in Table 2 and presented in Figs. 2, 3 and 4. Differential cross sections, $d \sigma / d \Delta \phi$, are presented as a function of the azimuthal angle difference, $\Delta \phi$, between the most forward jet and the scattered positron in bins of the variable $Y=\ln \left(x_{\mathrm{fwdjet}} / x\right)$. This variable approximates the rapidity distance between the scattered positron and the forward jet. For the selected data sample the normalised shape distributions, $1 / \sigma \cdot d \sigma / d \Delta \phi$, are also determined, where $\sigma$ is the integrated cross section in a given bin of $Y$. Furthermore, the forward jet cross section is measured as a function of $Y$.

The cross section, $d \sigma / d \Delta \phi$, as a function of $\Delta \phi$ is shown in Fig. 2 for three intervals of the variable $Y: 2.0 \leq Y<3.4$, $3.4 \leq Y<4.25$ and $4.25 \leq Y \leq 5.75$. These $Y$ bins correspond to average $x$ values of $0.0024,0.0012$ and 0.00048 , respectively. At higher values of $Y$ the forward jet is more decorrelated from the scattered positron. These results confirm with greater precision and more differentially the previous $\mathrm{H} 1$ measurement of the azimuthal correlation [11] which was performed in two different $x$ regions. 
Table 2 Differential forward jet cross section in bins of the variable $Y=\ln \left(x_{\mathrm{fwdjet}} / x\right)$ and the azimuthal angle difference $\Delta \phi$ between the most forward jet and the scattered positron. The statistical uncertainty $\left(\delta_{\text {stat }}\right)$, the uncertainty due to the hadronic energy scale $\left(\delta_{\text {had }}\right)$ and other systematic uncertainties $\left(\delta_{\text {syst }}\right)$ described in the text are given

\begin{tabular}{|c|c|c|c|c|}
\hline$\Delta \phi$ range $[\mathrm{rad}]$ & $d \sigma / d \Delta \phi[\mathrm{pb} / \mathrm{rad}]$ & $\delta_{\text {stat }}[\mathrm{pb} / \mathrm{rad}]$ & $\delta_{\text {had }}[\mathrm{pb} / \mathrm{rad}]$ & $\delta_{\text {syst }}[\mathrm{pb} / \mathrm{rad}]$ \\
\hline \multicolumn{5}{|l|}{$2.0 \leq Y<3.4$} \\
\hline $0.0-0.63$ & 27.3 & \pm 3.2 & $\begin{array}{l}+2.7 \\
-2.2\end{array}$ & $\begin{array}{l}+1.3 \\
-1.3\end{array}$ \\
\hline $0.63-1.26$ & 33.7 & \pm 3.4 & $\begin{array}{l}+2.8 \\
-3.3\end{array}$ & $\begin{array}{l}+1.9 \\
-1.8\end{array}$ \\
\hline $1.26-1.89$ & 35.8 & \pm 3.7 & $\begin{array}{l}+3.6 \\
-4.4\end{array}$ & $\begin{array}{l}+2.2 \\
-1.8\end{array}$ \\
\hline $1.89-2.51$ & 38.9 & \pm 3.8 & $\begin{array}{l}+4.4 \\
-4.4\end{array}$ & $\begin{array}{l}+2.3 \\
-2.6\end{array}$ \\
\hline $2.51-3.14$ & 47.9 & \pm 4.7 & $\begin{array}{l}+4.6 \\
-3.9\end{array}$ & $\begin{array}{l}+2.7 \\
-2.5\end{array}$ \\
\hline \multicolumn{5}{|l|}{$3.4 \leq Y<4.25$} \\
\hline $0.0-0.63$ & 48.2 & \pm 4.2 & $\begin{array}{l}+5.8 \\
-4.5\end{array}$ & $\begin{array}{l}+2.1 \\
-2.3\end{array}$ \\
\hline $0.63-1.26$ & 56.9 & \pm 4.3 & $\begin{array}{l}+6.1 \\
-6.4\end{array}$ & $\begin{array}{l}+2.8 \\
-2.6\end{array}$ \\
\hline $1.26-1.89$ & 58.7 & \pm 4.6 & $\begin{array}{l}+7.5 \\
-6.6\end{array}$ & $\begin{array}{l}+2.3 \\
-2.1\end{array}$ \\
\hline $1.89-2.51$ & 62.9 & \pm 4.8 & $\begin{array}{l}+6.3 \\
-6.6\end{array}$ & $\begin{array}{l}+2.6 \\
-3.1\end{array}$ \\
\hline $2.51-3.14$ & 60.4 & \pm 4.9 & $\begin{array}{l}+7.3 \\
-7.1\end{array}$ & $\begin{array}{l}+2.3 \\
-2.7\end{array}$ \\
\hline \multicolumn{5}{|l|}{$4.25 \leq Y<5.75$} \\
\hline $0.0-0.63$ & 55.1 & \pm 4.7 & $\begin{array}{l}+6.0 \\
-5.8\end{array}$ & $\begin{array}{l}+2.9 \\
-3.2\end{array}$ \\
\hline $0.63-1.26$ & 60.8 & \pm 5.0 & $\begin{array}{l}+5.2 \\
-6.7\end{array}$ & $\begin{array}{l}+2.6 \\
-2.9\end{array}$ \\
\hline $1.26-1.89$ & 60.0 & \pm 4.7 & $\begin{array}{l}+7.4 \\
-7.4\end{array}$ & $\begin{array}{l}+4.6 \\
-4.8\end{array}$ \\
\hline $1.89-2.51$ & 65.0 & \pm 5.4 & $\begin{array}{l}+7.7 \\
-7.3\end{array}$ & $\begin{array}{l}+4.2 \\
-4.1\end{array}$ \\
\hline $2.51-3.14$ & 57.3 & \pm 5.3 & $\begin{array}{l}+5.7 \\
-4.6\end{array}$ & $\begin{array}{l}+4.2 \\
-4.1\end{array}$ \\
\hline$Y$ range & $d \sigma / d Y[\mathrm{pb}]$ & $\delta_{\text {stat }}[\mathrm{pb}]$ & $\delta_{\text {had }}[\mathrm{pb}]$ & $\delta_{\text {syst }}[\mathrm{pb}]$ \\
\hline $2.00-3.25$ & 67.9 & \pm 3.3 & $\begin{array}{l}+7.5 \\
-7.7\end{array}$ & $\begin{array}{l}+3.0 \\
-3.1\end{array}$ \\
\hline $3.25-4.00$ & 194.4 & \pm 6.3 & $\begin{array}{l}+21.1 \\
-20.0\end{array}$ & $\begin{array}{l}+8.3 \\
-8.7\end{array}$ \\
\hline $4.00-4.75$ & 198.2 & \pm 6.7 & $\begin{array}{l}+22.5 \\
-23.4\end{array}$ & $\begin{array}{l}+10.4 \\
-10.4\end{array}$ \\
\hline $4.75-5.75$ & 92.3 & \pm 4.8 & $\begin{array}{l}+9.7 \\
-8.3\end{array}$ & $\begin{array}{l}+7.2 \\
-7.3\end{array}$ \\
\hline
\end{tabular}

The predictions of three QCD-based models with different underlying parton dynamics, discussed in Sect. 2, are compared with the data. The cross sections are well described in shape and normalisation by CDM which has a BFKL-like approach. In comparison to the older measurement the updated version of the CDM simulation with tuned parameters (see Sect. 2) shows slightly better agreement with the data. Predictions of RAPGAP, which implements DGLAP evolution, fall below the data, particularly at large $Y$. Calculations in the CCFM scheme as implemented in CASCADE using the UPDF set A0 [28] overestimate the measured cross section for large $\Delta \phi$ values in the two lowest $Y$ intervals. However, this model provides as good a description as CDM of the data in the highest $Y$ interval.

The shape of the $\Delta \phi$ distributions, $1 / \sigma \cdot d \sigma / d \Delta \phi$, is compared to the different MC predictions in the lower part of Fig. 2, where the ratio $R$ is shown, defined as:

$R=\left(\frac{1}{\sigma^{\mathrm{MC}}} \frac{d \sigma^{\mathrm{MC}}}{d \Delta \phi}\right) /\left(\frac{1}{\sigma^{\text {data }}} \frac{d \sigma^{\text {data }}}{d \Delta \phi}\right)$.
The precision of the measurements is shown at $R=1$ where the statistical and systematic uncertainties are indicated. The systematic uncertainty is reduced in the ratio and contains only two components added in quadrature: the model dependence of the correction factors and the trigger efficiency uncertainty. The ratio plots show that in the analysed phase space region the shape of the $\Delta \phi$ distributions is well described by all MC models. Since the shape predictions of the three models are very similar, this observable alone cannot discriminate among the models. It should be noted that the shape of the $\Delta \phi$ distributions is rather insensitive to the PDF used for event generation. The shape distributions generated using CTEQ6L, CTEQ6M [51] and HERAPDF1.0 [37] differ on average by $1-2 \%$. However, the cross section normalisation is more sensitive to the choice of PDF with differences up to $5 \%$ for CDM and up to $20 \%$ for RAPGAP at large $Y$.

Predictions of the CCFM model presented in Fig. 3 indicate a significant sensitivity to the choice of the uPDF. Set 


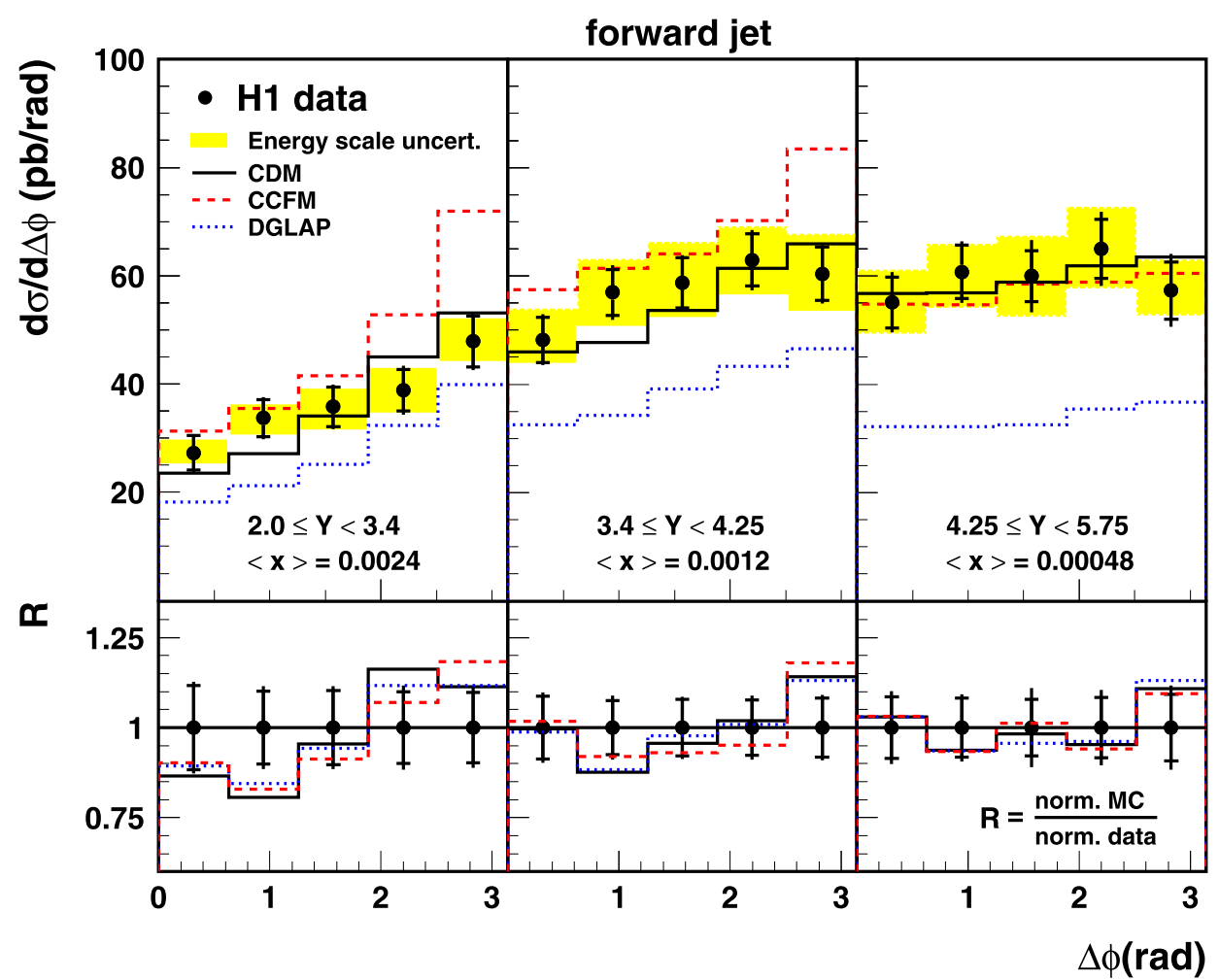

Fig. 2 Differential forward jet cross section as a function of the azimuthal angle difference $\Delta \phi$ between the most forward jet and scattered positron in three intervals of the variable $Y=\ln \left(x_{\mathrm{fwdjet}} / x\right)$. The inner error bars denote the statistical uncertainties. The systematic error due to the uncertainty of the hadronic energy scale is shown separately as a band around the data points. Other systematic uncertainties added quadratically to the statistical uncertainties are represented by the outer error bars. The data are compared with the predictions of DJANGOH/ARIADNE (CDM) and RAPGAP (DGLAP) with HERAPDF1.0 and with the CASCADE predictions (CCFM) which are shown with uPDF set A0. In the lower part of the figure the ratio $R$ of MC to data for normalised cross sections is shown. The precision of the measurements is shown at $R=1$ with the statistical and reduced systematic uncertainties indicated as error bars
Fig. 3 Differential forward jet cross section as a function of the azimuthal angle difference $\Delta \phi$ between the most forward jet and the scattered positron in three intervals of the variable $Y=\ln \left(x_{\mathrm{fwdjet}} / x\right)$. The data are compared to the predictions of CASCADE (CCFM) with two different sets of unintegrated gluon densities. For other details see caption to Fig. 2

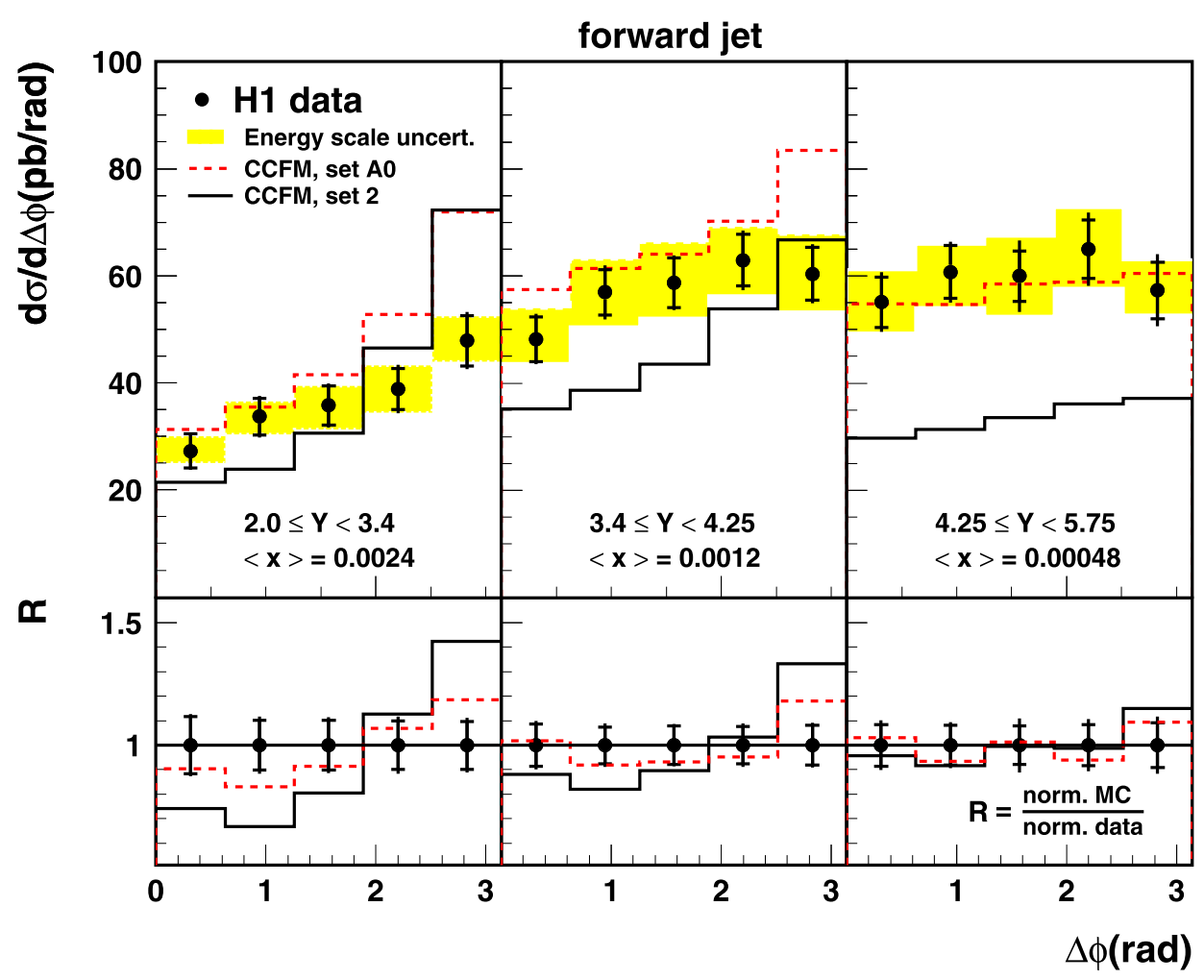


A0 and J2003-set 2 give quite different predictions for the differential cross sections in all $Y$ intervals. Set A0 provides a reasonable description of the measured cross sections, except for the region of large $\Delta \phi$ in the two lowest $Y$ bins. Predictions using J2003-set 2 do not describe the data, especially at higher $Y$, where the estimated cross sections are too low. The shape of the $\Delta \phi$ distributions is reasonably well described by the set A0. At low $Y$ it shows sensitivity to the unintegrated gluon density.

The cross section $d \sigma / d Y$ as a function of the rapidity separation $Y$ is shown in Fig. 4. The CDM model describes the data well over the whole $Y$ range. The DGLAP predictions fall below the data, but approach them at small $Y$. The predictions of the CCFM model are above the data at small $Y$ but describe them well at larger $Y$ corresponding to low values of $x$.

The forward and central jet cross sections and their uncertainties are given in Table 3 . The differential cross section $d \sigma / d \Delta \phi$ as a function of the azimuthal angle difference $\Delta \phi$ is shown in Fig. 5 in comparison with the predictions of the three MC models. The cross sections are measured in two intervals of $Y, 2.0 \leq Y<4.0$ and $4.0 \leq Y \leq 5.75$.

From Fig. 5 it is observed that at lower $Y$ the predictions of all models describe the cross sections reasonably well. At high $Y$ all models undershoot the data: CCFM (set A0) is

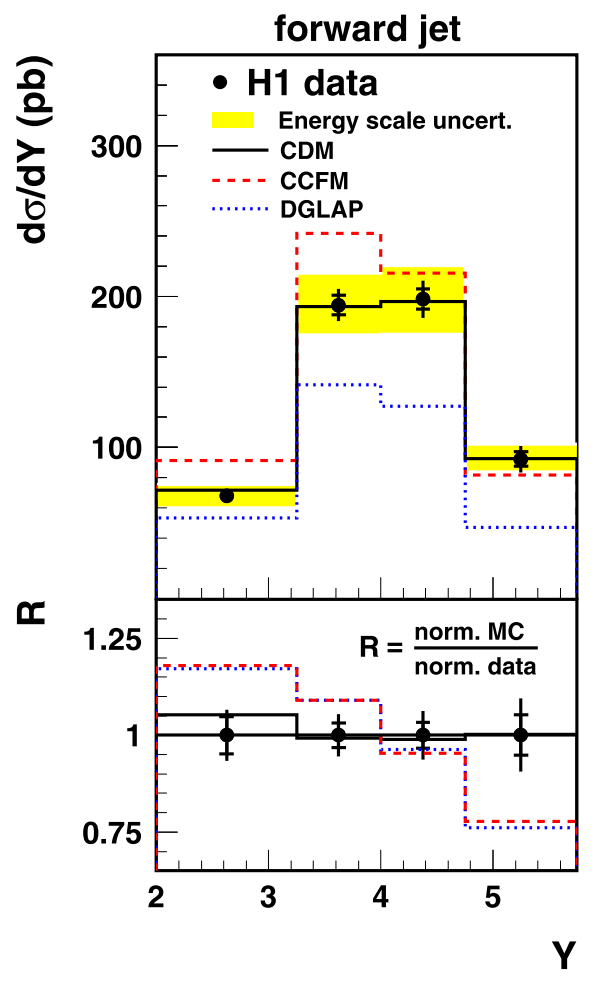

Fig. 4 Differential forward jet cross section as a function of the variable $Y=\ln \left(x_{\mathrm{fwdjet}} / x\right)$. The data are compared with the predictions of DJANGOH/ARIADNE (CDM) and RAPGAP (DGLAP) with HERAPDF1.0 and with the CASCADE predictions (CCFM) which are shown with uPDF set A0. For other details see caption to Fig. 2
Table 3 Differential forward and central jet cross section in bins of the variable $Y=\ln \left(x_{\mathrm{fwdjet}} / x\right)$ and the azimuthal angle difference $\Delta \phi$ between the most forward jet and the scattered positron. The statistical uncertainty $\left(\delta_{\text {stat }}\right)$, the uncertainty due to the hadronic energy scale $\left(\delta_{\text {had }}\right)$, and other systematic uncertainties $\left(\delta_{\text {syst }}\right)$ described in the text are given

\begin{tabular}{|c|c|c|c|c|}
\hline $\begin{array}{l}\Delta \phi \text { range } \\
{[\mathrm{rad}]}\end{array}$ & $\begin{array}{l}d \sigma / d \Delta \phi \\
{[\mathrm{pb} / \mathrm{rad}]}\end{array}$ & $\begin{array}{l}\delta_{\text {stat }} \\
{[\mathrm{pb} / \mathrm{rad}]}\end{array}$ & $\begin{array}{l}\delta_{\text {had }} \\
{[\mathrm{pb} / \mathrm{rad}]}\end{array}$ & $\begin{array}{l}\delta_{\text {syst }} \\
{[\mathrm{pb} / \mathrm{rad}]}\end{array}$ \\
\hline \multicolumn{5}{|c|}{$2.0 \leq Y<4.0$} \\
\hline $0.0-0.63$ & 18.9 & \pm 2.6 & $\begin{array}{l}+1.9 \\
-1.3\end{array}$ & $\begin{array}{l}+1.3 \\
-1.1\end{array}$ \\
\hline $0.63-1.26$ & 28.5 & \pm 2.9 & $\begin{array}{l}+2.3 \\
-2.9\end{array}$ & $\begin{array}{l}+2.0 \\
-1.9\end{array}$ \\
\hline $1.26-1.89$ & 31.6 & \pm 3.4 & $\begin{array}{l}+3.9 \\
-3.8\end{array}$ & $\begin{array}{l}+1.9 \\
-1.9\end{array}$ \\
\hline $1.89-2.51$ & 32.1 & \pm 3.2 & $\begin{array}{l}+3.6 \\
-2.7\end{array}$ & $\begin{array}{l}+1.3 \\
-1.3\end{array}$ \\
\hline $2.51-3.14$ & 33.9 & \pm 3.5 & $\begin{array}{l}+2.3 \\
-3.4\end{array}$ & $\begin{array}{l}+2.1 \\
-2.1\end{array}$ \\
\hline \multicolumn{5}{|c|}{$4.0 \leq Y<5.75$} \\
\hline $0.0-0.63$ & 39.5 & \pm 3.6 & $\begin{array}{l}+4.3 \\
-3.3\end{array}$ & $\begin{array}{l}+1.6 \\
-1.9\end{array}$ \\
\hline $0.63-1.26$ & 40.8 & \pm 3.6 & $\begin{array}{l}+3.4 \\
-3.9\end{array}$ & $\begin{array}{l}+2.2 \\
-2.2\end{array}$ \\
\hline $1.26-1.89$ & 41.8 & \pm 3.7 & $\begin{array}{l}+4.6 \\
-4.1\end{array}$ & $\begin{array}{l}+1.8 \\
-1.8\end{array}$ \\
\hline $1.89-2.51$ & 43.1 & \pm 4.1 & $\begin{array}{l}+5.2 \\
-4.4\end{array}$ & $\begin{array}{l}+2.2 \\
-2.2\end{array}$ \\
\hline $2.51-3.14$ & 34.9 & \pm 3.7 & $\begin{array}{l}+4.0 \\
-3.7\end{array}$ & $\begin{array}{l}+1.7 \\
-1.7\end{array}$ \\
\hline
\end{tabular}

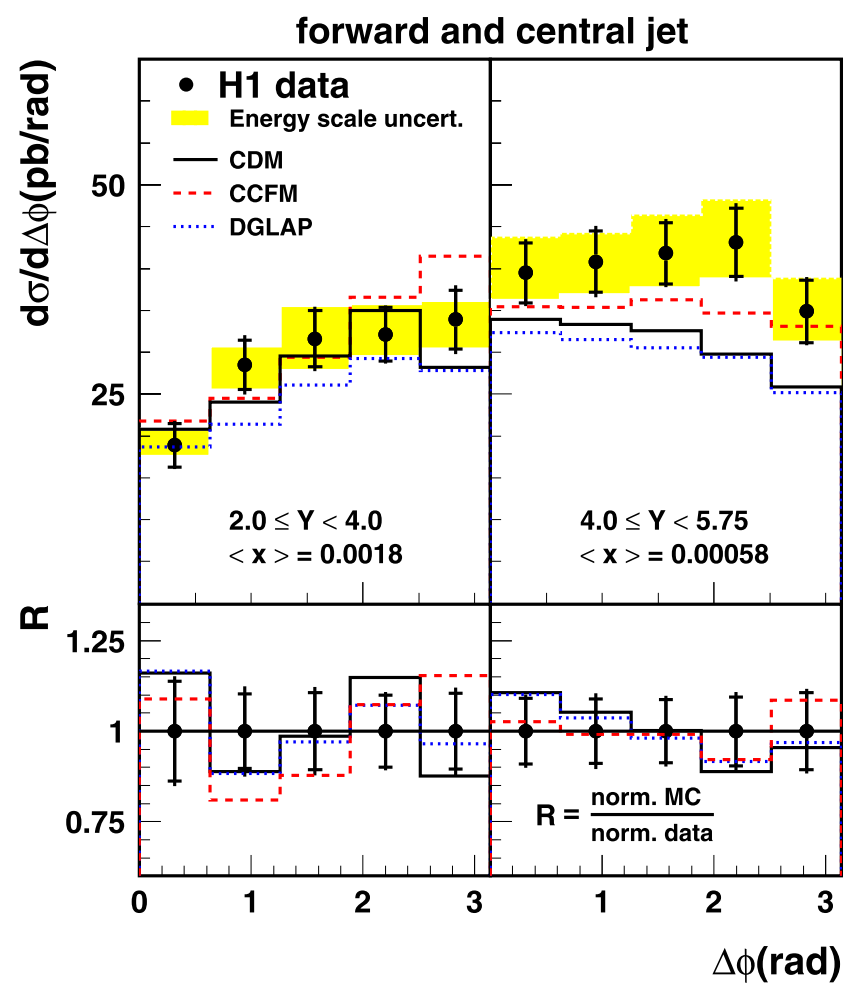

Fig. 5 Differential forward and central jet cross section as a function of the azimuthal angle difference $\Delta \phi$ between the most forward jet and the scattered positron in two intervals of the variable $Y=\ln \left(x_{\text {fwdjet }} / x\right)$. The data are compared with the predictions of DJANGOH/ARIADNE (CDM) and RAPGAP (DGLAP) with HERAPDF1.0 and with the CASCADE predictions (CCFM) which are shown with uPDF set A0. For other details see caption to Fig. 2 
Fig. 6 Differential forward jet cross section as a function of the azimuthal angle difference $\Delta \phi$ between the most forward jet and the scattered positron in three intervals of the variable $Y=\ln \left(x_{\text {fwdjet }} / x\right)$. NLO QCD predictions from NLOJET++ based on the CTEQ6.6 PDF, corrected to the level of stable hadrons, are shown as a solid line. Dashed lines above and below the nominal NLO prediction show the theoretical uncertainty estimated by applying a factor 2 or $1 / 2$ to the renormalisation and factorisation scales simultaneously. For other details see caption to Fig. 2

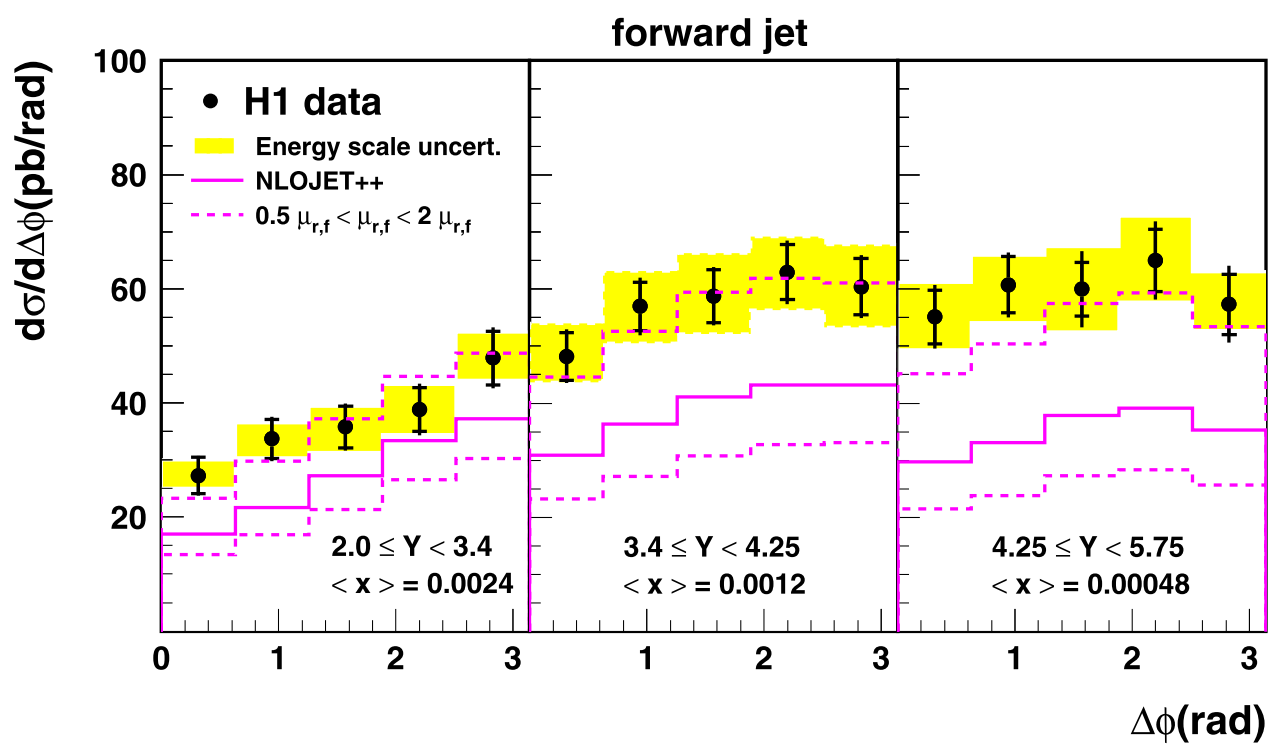

closest to the data, the DGLAP and CDM predictions are below the measured cross section. The ratio $R$ in the lower part of Fig. 5 shows that the shape of the $\Delta \phi$ distributions is well described by all MC models, as in the case of the forward jet measurements.

Comparisons of the measured $\Delta \phi$ distributions with NLOJET++ predictions are shown in Figs. 6 and 7. The calculations are performed at $O\left(\alpha_{S}^{2}\right)$ precision using the

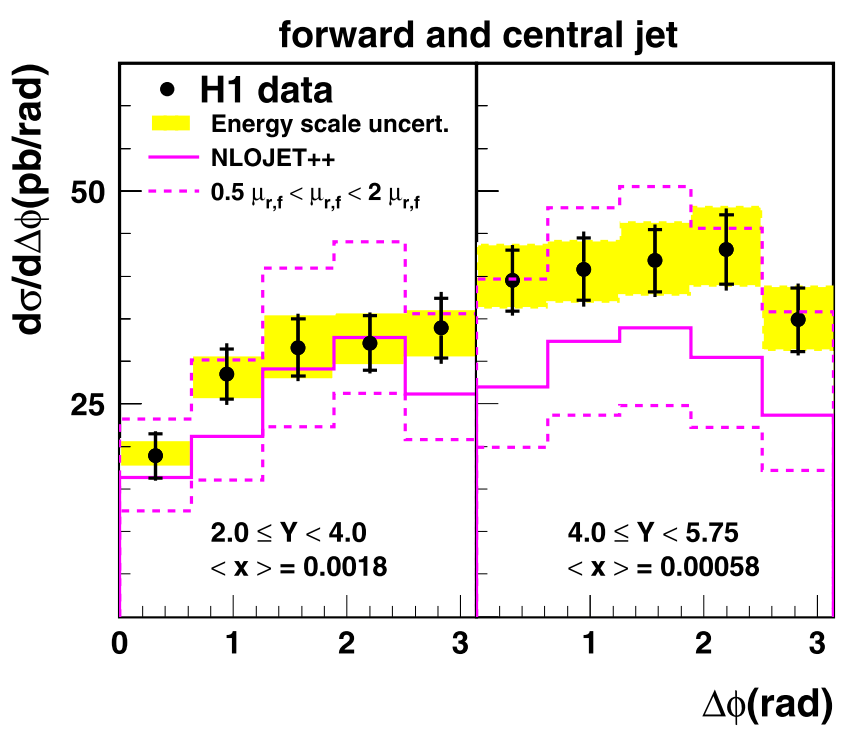

Fig. 7 Differential forward and central jet cross section as a function of the azimuthal angle difference $\Delta \phi$ between the most forward jet and the scattered positron in two intervals of the variable $Y=\ln \left(x_{\mathrm{fwdjet}} / x\right)$. NLO QCD predictions from NLOJET++ based on the CTEQ6.6 PDF, corrected to the level of stable hadrons, are shown as a solid line. Dashed lines above and below the nominal NLO prediction show the theoretical uncertainty estimated by applying a factor 2 or $1 / 2$ to the renormalisation and factorisation scales simultaneously. For other details see caption to Fig. 2
CTEQ6.6 PDF [41] and $\alpha_{S}\left(M_{Z}\right)=0.118$. Large theoretical uncertainties of up to $50 \%$ from the variation of factorisation and renormalisation scales are observed. The size of the theoretical uncertainty indicates that in this phase space region higher order contributions are expected to be important.

In the forward jet sample (Fig. 6) at lower $Y$ the data are above the central NLO result but still within the theoretical uncertainty. At highest $Y$, taking into account the systematic error of the data due to the uncertainty of the hadronic energy scale, the agreement between the data and theoretical predictions is marginal. In the case of the 'forward and central jet' sample shown in Fig. 7, the NLO calculation provides a reasonable description of the data at low $Y$. Only at high $Y$ in the regime of the BFKL evolution it is below the data, but again within the large theoretical uncertainty.

In summary, the correlation between the forward jet and the positron decreases with $Y$ and the $\Delta \phi$ distributions are flat at high $Y$. The measurements of the forward jet cross sections favour CDM and disfavour the RAPGAP model. CASCADE provides a reasonable description of the data at large $Y$, but shows sizeable sensitivity to the uPDF. The shape of the measured $\Delta \phi$ distributions is well described by MC models based on different QCD evolution schemes.

The similarity of the $\Delta \phi$ shapes of the MC predictions suggests that the forward jet predominantly originates from the hard matrix elements which are similar in all three models. However, MC studies with RAPGAP show that $80 \%$ of the forward jets are produced by parton showers. When the initial state parton shower is switched off, the shape of the $\Delta \phi$ distribution is only slightly changed, but the normalisation is significantly reduced. This indicates that the decorrelation in $\Delta \phi$ is mainly governed by the phase space requirements, in particular by the rapidity separation, $Y$, and that 
the normalisation of the cross sections is mainly influenced by the amount of soft radiation from parton showers, which depends on the evolution scheme.

\section{Conclusions}

Measurements of DIS events at low $Q^{2}$ containing a jet with high transverse momentum, produced in the forward direction at small angles with respect to the proton beam, are presented. Differential cross sections and normalised distributions are measured as a function of the azimuthal angle difference, $\Delta \phi$, and the rapidity separation, $Y$, between the forward jet and the scattered positron. Investigations of the azimuthal correlation between the most forward jet and the outgoing positron are performed in different regions of $Y$ for the forward jet sample and for the subsample with an additional central jet. To test the sensitivity of the measured observables to QCD dynamics at low $x$, the data are compared to QCD models with different parton evolution approaches and to predictions of next-to-leading order QCD calculations.

Measurements of the cross sections as a function of $\Delta \phi$ and $Y$ are best described by the BFKL-like CDM model, while the DGLAP-based RAPGAP model is substantially below the data. The CCFM-based CASCADE provides a reasonable description of the data but shows sizeable sensitivity to the unintegrated gluon density. The shape of the $\Delta \phi$ distributions does not discriminate further between different evolution schemes. The fixed order NLO DGLAP predictions are in general below the data, but still in agreement within the large theoretical uncertainties.

\begin{abstract}
Acknowledgements We are grateful to the HERA machine group whose outstanding efforts have made this experiment possible. We thank the engineers and technicians for their work in constructing and maintaining the $\mathrm{H} 1$ detector, our funding agencies for financial support, the DESY technical staff for continual assistance and the DESY directorate for support and for the hospitality which they extend to the non-DESY members of the collaboration.
\end{abstract}

Open Access This article is distributed under the terms of the Creative Commons Attribution Noncommercial License which permits any noncommercial use, distribution, and reproduction in any medium, provided the original author(s) and source are credited.

\section{References}

1. V. Gribov, L. Lipatov, Sov. J. Nucl. Phys. 15, 438 (1972)

2. V. Gribov, L. Lipatov, Sov. J. Nucl. Phys. 15, 675 (1972)

3. L. Lipatov, Sov. J. Nucl. Phys. 20, 94 (1975)

4. G. Altarelli, G. Parisi, Nucl. Phys. B 126, 298 (1977)

5. Y. Dokshitzer, Sov. Phys. JETP 46, 641 (1977)

6. E. Kuraev, L. Lipatov, V. Fadin, Sov. Phys. JETP 44, 443 (1976)

7. E. Kuraev, L. Lipatov, V. Fadin, Sov. Phys. JETP 45, 199 (1977)
8. Y. Balitsky, L. Lipatov, Sov. J. Nucl. Phys. 28, 822 (1978)

9. A.H. Mueller, Nucl. Phys. B, Proc. Suppl. 18C, 125 (1990)

10. S. Aid et al. (H1 Collaboration), Phys. Lett. B 356, 118 (1995). hep-ex/9506012

11. C. Adloff et al. (H1 Collaboration), Nucl. Phys. B 538, 3 (1999). hep-ex/9809028

12. A. Aktas et al. (H1 Collaboration), Eur. Phys. J. C 46, 27 (2006). hep-ex/0508055

13. J. Breitweg et al. (ZEUS Collaboration), Eur. Phys. J. C 6, 239 (1999). hep-ex/9805016

14. J. Breitweg et al. (ZEUS Collaboration), Phys. Lett. B 474, 223 (2000). hep-ex/9910043

15. S. Chekanov et al. (ZEUS Collaboration), Phys. Lett. B 632, 13 (2006). hep-ex/0502029

16. S. Chekanov et al. (ZEUS Collaboration), Eur. Phys. J. C 52, 515 (2007). arXiv:0707.3093

17. J. Bartels et al., Phys. Lett. B 384, 300 (1996). hep-ph/9604272

18. A. Sabio Vera, F. Schwennsen, Phys. Rev. D 77, 014001 (2008). arXiv:0708.0549

19. H. Jung, Comput. Phys. Commun. 86, 147 (1995) (see also http:// www.desy.de/ jung/rapgap.html). RAPGAP 3.1

20. K. Charchula, G.A. Schuler, H. Spiesberger, Comput. Phys. Commun. 81, 381 (1994). DJANGOH 1.4

21. L. Lönnblad, Comput. Phys. Commun. 71, 15 (1992). ARIADNE 4.10

22. A. Knutsson, Forward jet production in $e p$-collisions at HERA. Ph.D. thesis, Lund University, LUNFD6/(NFFL-7225) (2007) (http://www-h1.desy.de/publications/theses_list.html)

23. H. Jung, Comput. Phys. Commun. 143, 100 (2002)

24. H. Jung et al., Eur. Phys. J. C 70, 1237 (2010). CASCADE 2.2

25. M. Ciafaloni, Nucl. Phys. B 296, 49 (1988)

26. S. Catani, F. Fiorani, G. Marchesini, Phys. Lett. B 234, 339 (1990)

27. S. Catani, F. Fiorani, G. Marchesini, Nucl. Phys. B 336, 18 (1990)

28. H. Jung, in Proceedings of the XII International Workshop on Deep Inelastic Scattering (DIS2004), Štrbské Pleso, Slovakia, ed. by D. Bruncko, J. Ferencei, P. Stríženec (2004), p. 299. hep-ph/ 0411287

29. M. Hansson, H. Jung, in Proceedings of the XI International Workshop on Deep Inelastic Scattering (DIS2003), St. Petersburg, Russia, ed. by V.T. Kim, L.N. Lipatov (2003), p. 488. hep-ph/0309009

30. S. Aid et al. (H1 Collaboration), Nucl. Phys. B 470, 3 (1996). hep-ex/9603004

31. C. Adloff et al. (H1 Collaboration), Eur. Phys. J. C 21, 33 (2001). hep-ex/0012053

32. M. Derrick et al. (ZEUS Collaboration), Z. Phys. C 72, 399 (1996). hep-ex/9607002

33. S. Chekanov et al. (ZEUS Collaboration), Eur. Phys. J. C 21, 443 (2001). hep-ex/0105090

34. T. Sjöstrand, Comput. Phys. Commun. 82, 174 (1994). hep-ph/ 9508391. PYTHIA 5.7 and JETSET 7.4

35. T. Sjöstrand, Comput. Phys. Commun. 135, 238 (2001). PYTHIA 6.1

36. I.G. Knowles et al., in Workshop on Physics at LEP2, Geneva, Switzerland, ed. by G. Altarelli, T. Sjöstrand, F. Zwirner (1995), p. 103. CERN 96-01. hep-ph/9601212

37. F. Aaron et al. (H1 and ZEUS Collaborations), J. High Energy Phys. 1001, 109 (2010). arXiv:0911.0884

38. A. Kwiatkowski, H. Spiesberger, H.J. Möhring, Comput. Phys. Commun. 69, 155 (1992)

39. R. Brun et al., GEANT3. Technical Report CERN-DD/EE-84-1 (1987)

40. Z. Nagy, Z. Trocsanyi, Phys. Rev. Lett. 87, 82001 (2001). NLO$\mathrm{JET}++$ 
41. P. Nadolsky et al., Phys. Rev. D 78, 013004 (2008). arXiv: 0802.0007

42. I. Abt et al. (H1 Collaboration), Nucl. Instrum. Methods Phys. Res., Sect. A, Accel. Spectrom. Detect. Assoc. Equip. 386, 310 (1997)

43. I. Abt et al. (H1 Collaboration), Nucl. Instrum. Methods Phys. Res., Sect. A, Accel. Spectrom. Detect. Assoc. Equip. 386, 348 (1997)

44. R.D. Appuhn et al. (H1 SpaCal Group), Nucl. Instrum. Methods Phys. Res., Sect. A, Accel. Spectrom. Detect. Assoc. Equip. 386, 397 (1997)

45. B. Andrieu et al. (H1 Calorimeter Group), Nucl. Instrum. Methods Phys. Res., Sect. A, Accel. Spectrom. Detect. Assoc. Equip. 336, 499 (1993)
46. B. Andrieu et al. (H1 Calorimeter Group), Nucl. Instrum. Methods Phys. Res., Sect. A, Accel. Spectrom. Detect. Assoc. Equip. 350, 57 (1994)

47. T. Nicholls et al. (H1 SpaCal Group), Nucl. Instrum. Methods Phys. Res., Sect. A, Accel. Spectrom. Detect. Assoc. Equip. 374, 149 (1996)

48. C. Adloff et al. (H1 Collaboration), Z. Phys. C 74, 221 (1997). hep-ex/9702003

49. S. Catani et al., Nucl. Phys. B 406, 187 (1993)

50. S. Catani, Y. Dokshitzer, B.R. Webber, Phys. Lett. B 285, 291 (1992)

51. J. Pumplin et al., J. High Energy Phys. 012, 074 (2002). hep-ph/0201195 